\title{
Keratocystic Odontogenic Tumour: Current concepts, theory and presentation of 2 contrasting cases.
}

\author{
Dr. Kaushal Shah ${ }^{1}$, Dr. Jagruti Mistry ${ }^{2}$, Dr. Rajesh Koppikar ${ }^{3}$, \\ Dr. Amol Karagir ${ }^{4}$ \\ ${ }^{1,4}$ (Department of Oral Medicine \& Radiology, Bharati Vidyapeeth Deemed University Dental College, Sangli, \\ Maharashtra, India.) \\ ${ }^{2}$ (Department of Maxillofacial Surgery, Yogita Dental College, Khed, Maharashtra, India) \\ ${ }_{3}^{3}$ (Department of Periodontology, Bharati Vidyapeeth Deemed University Dental College, Sangli, Maharashtra,
} India)

\begin{abstract}
Odontogenic keratocyst (OKC) is a cyst of tooth origin with an aggressive clinical behavior including a high recurrence rate. It has been rechristened to Keratocystic odontogenic tumour (KCOT), as it better reflects its neoplastic nature. We report 2 contrasting cases of KCOT in association with impacted teeth and revisit the various treatment modalities used to tackle the aggressive nature and keep its recurrence at bay. Most promising being use of molecular biology to treat such tumours which could eventually reduce or eliminate the need for aggressive methods to manage the lesions.
\end{abstract}

Keywords: benign, keratocystic Odontogenic tumor, , locally aggressive, Odontogenic keratocyst, , molecular biology

\section{Introduction}

First described by Philipsen in 1956, ${ }^{1}$ the odontogenic keratocyst (OKC) is now designated by the World Health Organization (WHO) as a keratocystic odontogenic tumour (KCOT) and is defined as "a benign uni- or multicystic, intraosseous tumour of odontogenic origin, with a characteristic lining of parakeratinized stratified squamous epithelium and potential for aggressive, infiltrative behaviour." ${ }^{2}$ WHO "recommends the term keratocystic odontogenic tumour as it better reflects its neoplastic nature." ${ }^{2}$

In 1967, Toller suggested that the OKC may best be regarded as a benign neoplasm rather than a conventional cyst based on its clinical behavior. ${ }^{3}$ In 1984, Ahlfors and others ${ }^{4}$ suggested that "if the OKC were recognized as a true, benign cystic epithelial neoplasia, the question of modified treatment schedules would be raised."

Harring et al. ${ }^{5}$ best characterized this cyst by stating that "After thirty years of study, questions related to the histogenesis, pathogenesis, histology, high recurrence rate, and neoplastic potential of the OKC are still being debated."

In the years since, published reports have influenced WHO to reclassify the lesion as a tumour. Several factors form the basis of this decision.

- Behaviour: As described earlier, the KCOT is locally destructive and highly recurrent.

- Histopathology: Studies such as that by Ahlfors and others ${ }^{4}$ show the basal layer of the KCOT budding into connective tissue. In addition, WHO notes that mitotic figures are frequently found in the suprabasal layers. ${ }^{2}$

- Genetics: PTCH ("patched"), a tumour suppressor gene involved in both NBCCS and sporadic KCOTs, normally forms a receptor complex with oncogene SMO ("smoothened") for the SHH ("sonic hedgehog") ligand. PTCH binding to SMO inhibits growth-signal transduction. SHH binding to PTCH releases this inhibition. ${ }^{6}$ If normal functioning of PTCH is lost, the proliferation-stimulating effects of SMO are permitted to predominate.

In light of the reclassification, it is appropriate to review the salient features of this well-known lesion and to consider the implications for treatment.

\section{Case Presentations}

2.1 Case 1. A 34 year-old patient reported to the Department of Oral Medicine and Radiology, with a chief complaint of pain \& swelling over the right side of the face since $1 \frac{1}{2}$ months associated with pus discharge. Extra-2.1.1 Oral examination revealed presence of a diffuse swelling over the right ramus of mandible, The swelling was hard in consistency, however no lymph nodes were palpable. Intra- Oral Examination showed a diffuse swelling with respect to mandibular right first and second molar, obliterating the 
buccal vestibule. (Fig.1) Swelling was hard in consistency on palpation. The associated teeth showed no mobility.

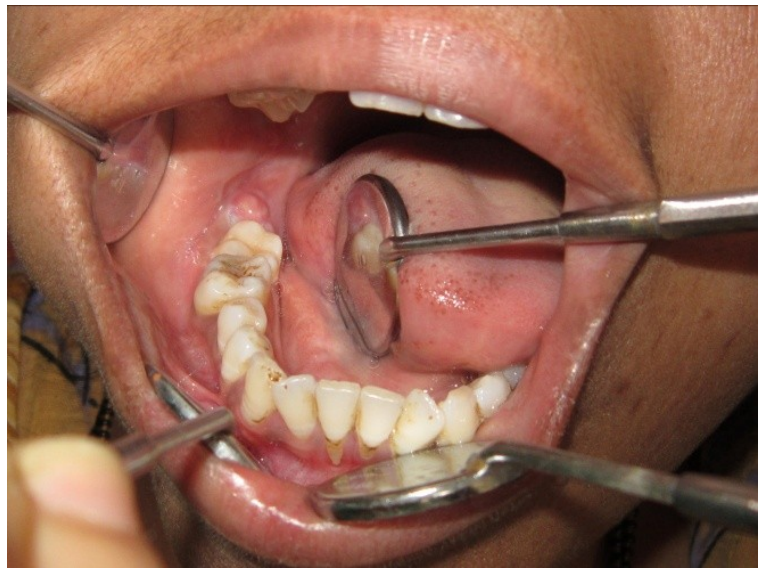

Fig.1 Intra-oral photograph

2.1.2 Radiographic Investigations An Orthopantomograph(OPG) revealed a well corticated unilocular radiolucency with scalloped margins in the right ramus of mandible extending upto distal root of the second molar. An impacted third molar was visible, however there was no sign of displacement of the teeth or resorption of roots.(figure 2)

-Computed Tomographic (CT) axial scan showed an expansile, osteolytic lesion in the right ramus of the mandible (figure 3)

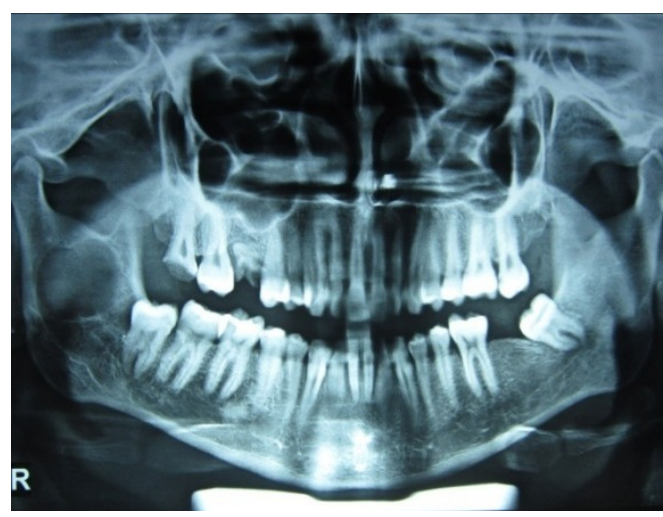

Fig.2 Orthopantomograph

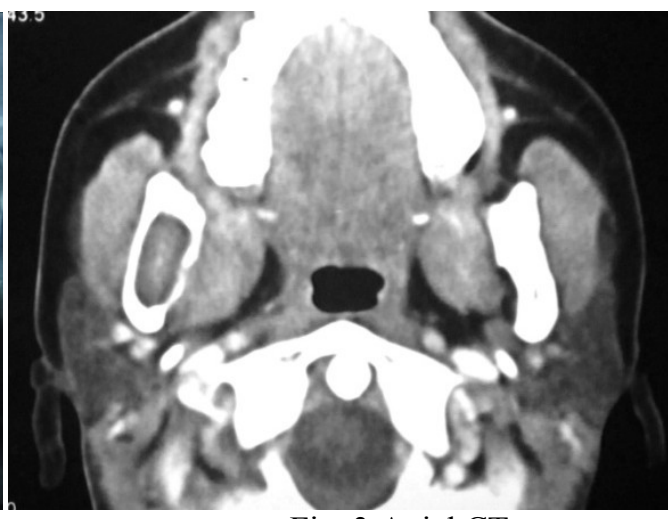

Fig. 3 Axial CT

2.1.3Histopathologic Investigation : Biopsy of the surgically enucleated lining(figure 4) showed keratinized squamous epithelium, lack of rete ridges and an artifactual separation from the basement membrane. Histopathological Diagnosis : Odontogenic Keratocyst

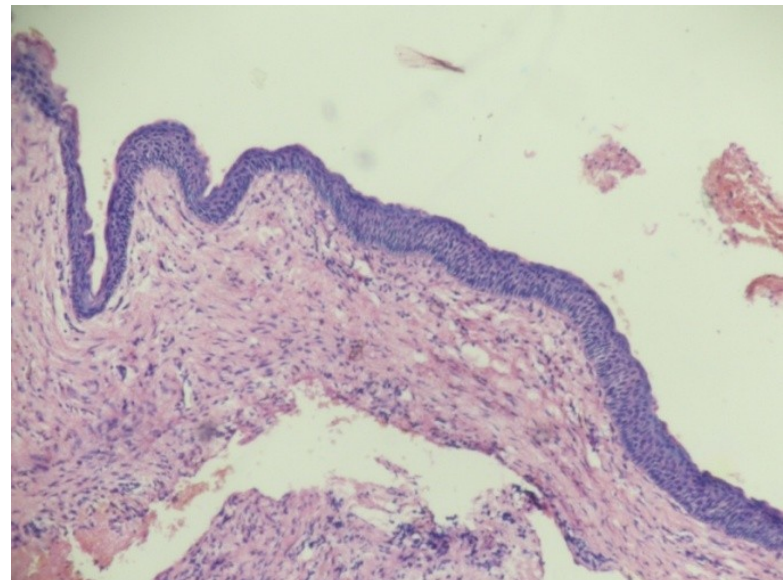

Fig.4 H \& E Photomicrograph(10x magnification) 
2.2 Case 2 : A 20 year old patient came with a chief complaint of gradual increase in swelling on right side of face since 2 months.

2.2.1 Examination revealed a diffuse swelling over right maxilla, extra-orally (figure 5). It was hard in consistency and slightly tender on palpation. (Fig. 5)

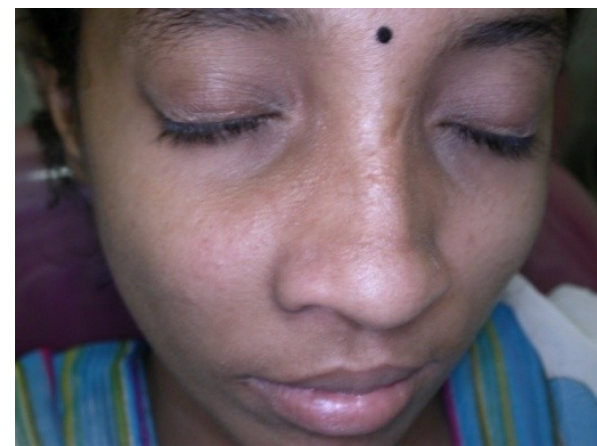

Fig.5 Extra-oral photograph

\subsubsection{Radiographic Investigations}

An Intra-oral periapical (IOPA)(Fig.6) and Orthopantomograph (OPG) (Fig.7) revealed an extensive corticated radiolucency showing scalloped margins associated with an impacted supernumerary tooth. The lesion involved the right maxillary sinus, extending from the incisors upto the molars, causing some displacement of the lateral incisor and canine.

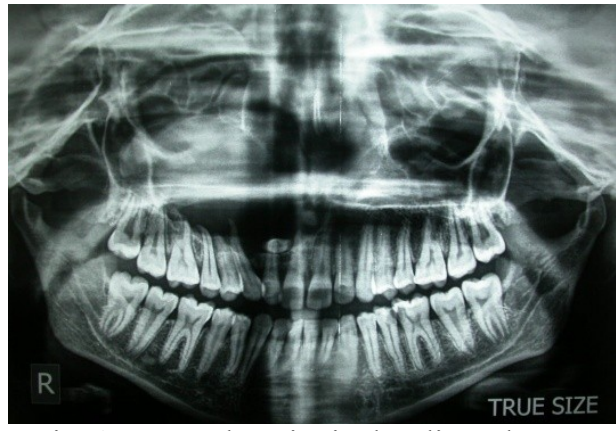

Fig.6 Intraoral Periapical radigraph

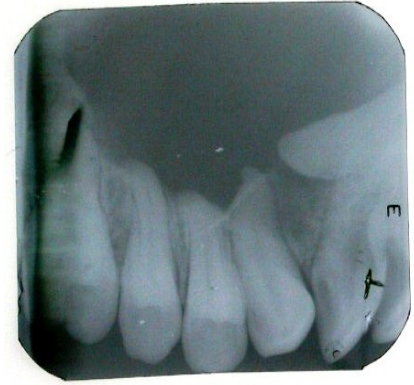

Fig.7Orthopantomograph

-A Waters projection showed opacification of the right maxillary sinus and right side nasal cavity. However no deviation of the nasal septum was noted.(Fig.8)

-A Computed Tomgraphic (CT) coronal slice revealed an expansile osteolytic lesion associated with an impacted tooth, obliterating the right maxillary sinus and inferior and middle meatus and conchae.(Fig. 9)

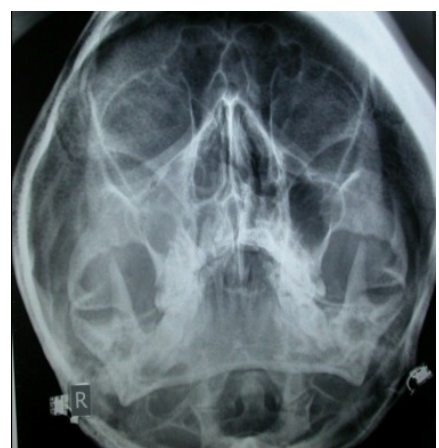

Fig.8 PA Water's View

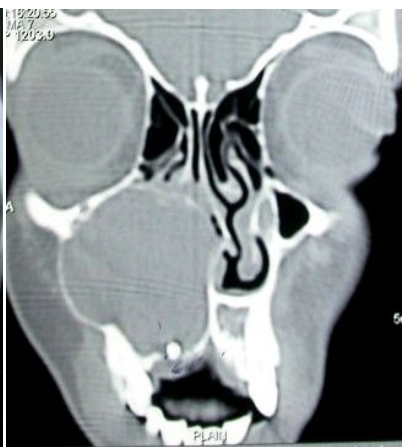

Fig.9 Coronal CT

\section{Treatment}

Case 1 underwent Carnoy's solution fixation of the cystic wall before complete enucleation of the KCOT. We plan to use the same mode of treatment for Case 2, keeping in mind the age, extent of the lesion and proximity to vital structures. 


\section{Outcome and Follow-up}

Since resection of the jaw, which has the lowest chances of recurrence was not attempted, due to its radical nature, a long term follow-up is necessary. Patient has been advised for a yearly clinical and radiologic follow-up for minimum 5 years.

\section{Discussion}

KCOTs comprise approximately $11 \%$ of all cysts of the jaws. ${ }^{7}$ They occur most commonly in the mandible, especially in the posterior body and ramus regions., ${ }^{2,8}$ They almost always occur within bone, although a small number of cases of peripheral KCOT have been reported. ${ }^{10-12}$. Patients may present with swelling, pain and discharge or may be asymptomatic. Distinctive clinical features include a potential for local destruction and a tendency for multiplicity, especially when the lesion is associated with nevoid basal cell carcinoma syndrome (NBCCS) or Gorlin-Goltz syndrome. KCOTs have a high recurrence rate, reportedly between $25 \%$ and $60 \%{ }^{13}$

In 1976, Brannon ${ }^{9}$ proposed 3 mechanisms for KCOT recurrence: incomplete removal of the cyst lining, growth of a new KCOT from satellite cysts (or odontogenic rests left behind after surgery) and development of a new KCOT in an adjacent area that is interpreted as a recurrence. ${ }^{14}$

Morgan and colleagues ${ }^{15}$ categorize surgical treatment methods for KCOT as conservative or aggressive. Conservative treatment is "cyst-oriented" and, thus, includes enucleation, with or without curettage, or marsupialization. Its advantage is preservation of anatomical structures (including teeth), which is advocated because KCOTs commonly present in younger patients. It has been asserted that a conservative approach is applicable not only to all age groups, but also to patients with NBCCS. ${ }^{16}$ Aggressive treatment addresses the "neoplastic nature" of the KCOT and includes peripheral ostectomy, chemical curettage with Carnoy's solution or en bloc resection. Aggressive modalities have generally been recommended for NBCCS cases, large KCOTs and recurrent lesions. ${ }^{16} \mathrm{~A}$ review of the literature suggests that recurrence rate is relatively low with aggressive treatment, whereas more conservative methods tend to result in more recurrences ${ }^{17}$ (Table 1)

Table 1 : Summary of treatment related to recurrence rate

\begin{tabular}{|l|c|c|c|}
\hline \multicolumn{1}{|c|}{ Treatment } & Lesions & Recurrences & Recurrence rate; \\
\hline Enucleation & 465 & 141 & 30 \\
\hline $\begin{array}{l}\text { Enucleation + } \\
\text { Carnoy's solution }\end{array}$ & 122 & 11 & 9 \\
\hline $\begin{array}{l}\text { Enucleation + } \\
\text { Peripheral Ostectomy }\end{array}$ & 11 & 2 & 18 \\
\hline $\begin{array}{l}\text { Enucleation + } \\
\text { Carnoy's+ } \\
\text { Peripheral ostectomy }\end{array}$ & 83 & 7 & 8 \\
\hline $\begin{array}{l}\text { Enucleation + } \\
\text { cryotherapy }\end{array}$ & 29 & 11 & 38 \\
\hline $\begin{array}{l}\text { Marsupialization } \\
\text { Marsupialization + } \\
\text { cystectomy }\end{array}$ & 108 & 6 & 33 \\
\hline Resection & 39 & 14 & 13 \\
\hline
\end{tabular}

An excellent reference for various modalities is by Voorsmit and Stoelinga ${ }^{18}$ where multiple surgical approaches with and without the use of Carnoy's fixative solution is recommended. In brief, this study shows that Carnoy's solution is effective in reducing recurrence rate when patients were followed for two years.

In recent years, studies have hinted at possible new treatment methods for KCOT. According to Taipale and colleagues, ${ }^{19}$ cyclopamine, a plant-based steroidal alkaloid, inhibits the cellular response to the SHH signal.

They found that cyclopamine blocks activation of the SHH pathway caused by oncogenic mutation making it a potential "mechanism-based" therapeutic agent for human tumours whose pathogenesis involves excess SHH pathway activity. Zhang and others ${ }^{20}$ postulate that antagonists of SHH signaling factors could effectively treat KCOTs. Their suggested strategies include the reintroduction of a wild-type form of PTCH, inhibiting the SMO molecule by synthetic antagonists and suppressing the downstream transcription factors of the SHH pathway. They suggest that intracystic injection of an SMO protein-antagonist has the greatest potential as a future treatment option.

The aggressive nature of KCOT warrants an aggressive treatment strategy, and its recent reclassification by WHO as a neoplasm should further motivate clinicians in this direction. Resection of the jaw 
results in the lowest recurrence rate. However, considering the radical nature of the procedure, unless resection is necessary, it is acceptable to use enucleation in combination with Carnoy's solution (the approach we used to treat our case with success) or marsupialization and peripheral ostectomy.

As the entity is relatively rare, opinions among experts about how to treat KCOT's differ.

To summarize, the available treatment options ${ }^{17}$ are

- Wide en bloc surgical resection

- Enucleation-complete removal of cystic lining

- Marsupialization-the surgical opening of the cavity and a creation of a marsupial-like pouch, so that the cavity is in contact with the outside for an extended period, e.g. three months

- Curettage (simple excision and scrape-out of cavity)

- Simple excision

- Carnoy's solution (chemical fixation)-usually used in conjuction with excision or enucleation

- Enucleation and cryotherapy ${ }^{21}$

- Possible use of a molecular biologic agent such as cyclopamine for non aggressive mode of treatment ${ }^{19,20}$

\section{Conclusion} simple cyst

The aggressive nature of the KCOT underscores the fact that this lesion should not be managed as a

KCOT should be treated by resection, or a combination of enucleation with an adjunctive treatment like Carnoy's solution, so as to decrease the chances of recurrence. Though radical resection has no recurrence, it does have the highest morbidity rate and should be reserved for multiple recurrent cysts, after conservative combination treatment have failed. A long term clinical and radiologic follow-up is of paramount importance As research continues, treatment may become molecular in nature. This could eventually reduce or eliminate the need for aggressive methods to manage the lesions. Currently, the reclassification of OKC as a tumour and the research and understanding of the lesion that has influenced this change should serve as a reminder to deal with these entities with more concern and long term follow-up.

\section{References}

[1]. Philipsen HP. Om keratocystedr (Kolesteratomer) and kaeberne. Tandlaegebladet 1956;60:963-971.

[2] Barnes L, Eveson JW, Reichart P, Sidransky D, editors. Pathology and genetics of head and neck tumours. Lyon: IARC Press; 2005. WHO classification of tumours series.

[3] Toller P. Origin and growth of cysts of the jaws. Ann R Coll Surg Engl 1967;40(5):306-336.

[4] Ahlfors E, Larsson A, Sjögren S. The odontogenic keratocyst: a benign cystic tumor? J Oral Maxillofac Surg 1984;42(1):10-19.

[5] Haring JI, Van Dis ML. Odontogenic keratocysts; a clinical, radiographic and histopathologic study. Oral Surg Oral Med Oral Pathol 1988;66:145-153.

[6] Cohen MM. Nevoid basal cell carcinoma syndrome: molecular biology and new hypotheses. Int J Oral Maxillofac Surg 1999;28(3):216-223

[7] Maurette PE, Jorge J, de Moraes M. Conservative treatment protocol of odontogenic keratocyst: a preliminary study. J Oral Maxillofac Surg 2006;64(3):379-383.

[8] Browne RM. The odontogenic keratocyst: clinical aspects. Br Dent J 1970;128(5):225-231.

[9] Brannon RB. The odontogenic keratocyst. A clinicopathologic study of 312 cases. Part I. Clinical features. Oral Surg Oral Med Oral Pathol 1976;42(1):54-72.

[10] Dayan D, Buchner A, Gorsky M, Harel-Raviv M. The peripheral odontogenic keratocyst. Int J Oral Maxillofac Surg 1988; 17(2):81-3.

[11] Worrall SF. Recurrent odontogenic keratocyst within the temporalis muscle. Br J Oral Maxillofac Surg 1992;30(1):59-62.

[12] Chehade A, Daley TD, Wysocki GP, Miller AS. Peripheral odontogenic keratocyst. Oral Surg Oral Med Oral Pathol 1994; 77(5):494-497.

[13] Sapp JP, Eversole LR, Wysocki GP. Contemporary oral and maxillofacial pathology. 2nd ed. St. Louis: Mosby;2004,p54.

[14] Woolgar JA, Rippin JW, Browne RM. A comparative study of the clinical and histological features of recurrent and non-recurrent odontogenic keratocysts. J Oral Pathol 1987;16(3):124-128.

[15] Morgan TA, Burton CC, Qian F. A retrospective review of treatment of the odontogenic keratocyst. J Oral Maxillofac Surg 2005;63(5):635-639.

[16] Meiselman F. Surgical management of the odontogenic keratocyst: conservative approach. J Oral Maxillofac Surg 1994;52(9):960963.

[17] Madras J, Lapointe H. Keratocystic Odontogenic Tumour: Reclassification of the Odontogenic Keratocyst from Cyst to Tumour. J Can Dent Assoc. 2008 Mar;74(2):165-165h

[18] Voorsmit RACA, Stoelinga PJW, van Haelst UJGM. The management of keratocysts. J Maxillofac Surg 1981;9:228-236.

[19] Taipale J, Chen JK, Cooper MK, et al. Effects of oncogenic mutation in Smoothened and Patched can be reversed by cyclopamine. Nature 2000;406(6799):1005-1009.

[20] Zhang L, Sun ZJ, Zhao YF, Bian Z, Fan MW, Chen Z. Inhibition of SHH signaling pathway: molecular treatment strategy of odontogenic keratocyst. Med Hypotheses 2006;67(5):1242-1244. Epub 2006 June 27.

[21] Schmidt BL, Pogrel MA. "The use of enucleation and liquid nitrogen cryotherapy in the management of odontogenic keratocysts." J Oral Maxillofac Surg. 2001;59:720-727 